\title{
Towards an Intelligent Web of Things
}

\author{
Nafaâ Jabeur \\ Dept. of Computer Science \\ German University of Technology in Oman - GUtech \\ P.O. Box 1816, PC 130, Muscat, Oman \\ nafaa.jabeur@gutech.edu.om
}

\author{
Hedi Haddad \\ Dept. of Computer Science \\ Dhofar University \\ P.O. Box 2509, P.C 211, Dhofar, Oman \\ hhaddad@du.edu.om
}

\begin{abstract}
Although it is an important subset of the increasingly popular Internet of Things (IoT), the Web of Things (WoT) did not attract enough research and development attention in academia and business, worth of its value. We believe that this is due to its numerous challenges, especially when it comes to providing Web services to and from a myriad of heterogeneous devices with limited resources, random mobility, constrained network connections, and lack of standardized communication protocols. We argue that these devices should be augmented with artificial intelligence techniques for an enhanced management of their resources and an easier construction of Web applications integrating Real World Things (RWT). To this end, we present in this paper a new classification of the WoT challenges and highlight the opportunities of embedding smartness into RWT. We also present our vision of Intelligent WoT by proposing a multiagent system-based architecture for intelligent Web service composition. Finally, we discuss this architecture within the context of an Intelligent VANET-WoT scenario.
\end{abstract}

Index Terms-Internet of Things, Web of Things, Mutliagent Systems, Web service, Web service composition, VANET;

\section{INTRODUCTION}

In the recent decades, technological advances have brought communication and computing technologies from large to small and tiny scales. Wireless Sensor Networking (WSN) technologies have then arisen as promising tools where spatially distributed sensing devices are deployed to collect insitu data on a variety of spatial objects and events of interest, anytime and everywhere [26]. These devices are since there benefiting from incessant progress in the fields of miniaturization, networking capabilities, and mobile and pervasive computing. They are not anymore being considered as simple data collecting devices. They are now being endowed with processing and intelligent capabilities to assess on their own their current situations and make the right decision at the right time. A new era bridging cyber and physical worlds have then emerged with the vision to insert smartness everywhere. This era is particularly marked with the recent emergent fields of Cyber Physical Systems [25] and Internet of Things.

The Internet of Things (IoT) could be defined as a global networking infrastructure that uses data capturing devises and communication resources to link virtual and physical objects [1]. It can, therefore, be thought of as an integration of a variety of sensing, communication, and networking devices and systems in order to connect people and things with common interests, so that anybody can efficiently access the information of any object and any service, at any time and any place, regardless the heterogeneity of devices and communication protocols. [2]. The Web of Things (WoT) is a subset of the IoT where web standards are used to seamlessly integrate and connect physical objects and information resources [3]. The emerging breakthrough of WoT is expected to offer solutions in a wide variety of domains, including healthcare applications, military and rescue scenarios, energy monitoring, and transportation management. This is expected to be facilitated thanks to the increasing abundance of smart devices with webenabled capabilities.

The WoT vision particularly aims to use web protocols and technologies to allow the easy construction of web applications exploiting Real World Things (RWT). However, because of the heterogeneity of their hardware/software specification and capabilities as well as their data quality and requirement, along with their commonly non-deterministic mobility, RWT are facing serious problems to interoperate. These problems are increasingly challenging because of the absence of widely accepted standards. With the continuous expansion of cyber and physical words toward each other as well as toward a social world, additional challenges of trust, privacy and security are raising up.

As it can be seen clearly, the challenges of the WoT concern several levels and issues. We believe that autonomy, flexibility, and intelligence must be integrated to any approach addressing these challenges. We argue that techniques from the artificial intelligence field would allow for efficient candidate solutions. In this perspective, few approaches (e.g. [5,16]) have been proposed. However, the integration of intelligence into RWT has not been clearly investigated.

Furthermore, a major success factor for the WoT is driven by the prevalence of web expertise. The Internet networking infrastructure and the existing data representation, storage, visualization, and sharing standards are, indeed, pillars of the WoT vision. Nevertheless, these standards and techniques must be revised, extended, and/or revolutionized in order to meet the requirements of the RWT and allow them to integrate the Web and mutually exchange web services. These services should be easy to publish, discover, compose, and execute. The traditional web service paradigm should then be enriched by promoting the web from both cyber and physical worlds [3]. Because of their hardware and software limitations, it would be 
beneficial to the RWT to collaboratively provide services going beyond their individual capabilities. We then argue that these RWT should organize themselves into groups where Webenabled devices could be proxies to others to connect to the Internet and share their services.

As the issues of service composition and clustering within the context of WoT were not specifically investigated, we propose in this paper to address them as well as other challenges of the WoT using a multiagent-based approach. In the reminder of this paper, Section 2 highlights existing works that have addressed the issue of web service provision in the WoT. Section 3 proposes a categorization of the WoT challenges. Section 4 addresses the issue of intelligent WoT where the need for intelligent techniques are emphasized and explained in a scenario of Web of Vehicular Ad-Hoc Network.

\section{RELATED WORK}

The main challenge of the IoT and therefore the WoT is to allow a countless number of RWT to interoperate and "understand" each other. To facilitate this interoperability, several industry alliances and standards, including Universal Plug and Play (UPnP), DLNA, SLP, and Zeroconf have been defined [4]. Each of these standards has individually been successful in enabling devices to communicate with each other [5]. However, they are not strictly standardized and some of them are unsuitable to resource-constrained devices due to their heavy protocols. Thanks to the increasing integration of webenabled capabilities, large number of RWT are currently benefiting from the existent networking infrastructure of the Internet. The WoT is then providing these RWT with the application and service layer to interoperate over HTTP [6,7]. Other networking infrastructures like Wi-Fi and Ethernet allow for new opportunities to build additional applications and services [5]. Furthermore, with the decreasing size of embedded systems and their increasing hardware and software capabilities, it has become possible to integrate lightweight Web servers into many appliances [8]. This trend to use the Web as a platform to create and integrate applications that integrate RWT into the Web has attracted attention in academia and business [7,9]. This resulted in the increasing use of Web services for the interoperability of RWT, particularly because of their proprietary and heterogeneous technologies [5]. The possible integration of different heterogeneous RWT into the Web leads to a more advanced perspective, where these things are abstracted into reusable web services, and not only regarded as simple web pages [3]. For instance, RESTful APIs and SOAP-based Web Services (WS-*) allow RWT to offer their functionalities. RESTful Web services are based on Representational State Transfer (REST) [10] which is lightweight, simple, loosely coupled, flexible and seamlessly integrates into the Web using HTTP as an application protocol [5]. Although REST-based services are being incorporated into many WoT applications, particularly where Quality of Service (QoS) levels are strictly applied (e.g., banking and stock market), a more tightly coupled service paradigm like WS-* would be more ideal [11]. Recent developments are successfully allowing to embed tiny web servers into RWT (e.g., [12,22]), especially since these servers do not need to handle large number of simultaneous connections and request. However, a lot of research and development efforts remain necessary in order to appropriately manage the increasing volume of demands from these servers while preserving the limited resources of the corresponding RWT.

In the current literature, the WoT did not attract enough research and development attention, worth of its value. We believe that this is due to its numerous challenges as well as the lack of maturity of related communication and processing capabilities of RWT. We also believe that artificial intelligence techniques which have proven their extraordinary performance in dealing with problems of highly dynamic, uncertain, and heterogeneous environments could bring solutions to the problems of WoT. Some works have integrated such techniques within the context of IoT (e.g., [13,14,15]). However, to the best of our knowledge this was not the case for the WoT. An interesting study is proposed by Zhong et al. [16] where the authors have proposed a holistic intelligence methodology called Wisdom WoT (W2T) for realizing "the harmonious symbiosis of humans, computers, and things in the hyper world" [16]. The methodology particularly aims to implement a closed cycle that starts from things to data, information, knowledge, wisdom, services, humans, and then back to things. This macro-level cycle is not embedded on the RWT which are mostly being considered as data collectors with networking facilities to connect to server providers.

\section{Challenges of WoT}

Building the WoT basically concerns ways to design and implement scalable and industry-ready IoT solutions on the Web. As a subset of the IoT, the WoT shares many characteristics with Wireless Sensor Networking (WSN), Machine-to-Machine (M2M), and ubiquitous computing technologies. Furthermore, the WoT integrates physical and information objects, necessitating new means to model and reason about a range of context types [17]. From a design perspective and compared to the traditional server-client architecture, the WoT has a flat architecture, where two main challenges need to be considered: a) integrating the RWT to the web; and b) making the RWT provide composable and interoperable web services? [3]. From a general perspective, we classify the challenges of WoT into four main categories: Data Processing and Storage, Service Management, Networking and Communication, and Security, Privacy, and Trust (Figure 1).

Data Processing and Storage. The spatially distributed RWT, commonly moving in the space and collecting data, anytime, anywhere and for a variety of purposes, are basically facing problems to make the appropriate use of their data. In this regard, the RWT have to identify which data is important to collect for the current situation and according to which sampling frequency. The data collected should then be filtered and evaluated according to its semantics, the current context as well as current and expected requirements. The next challenge is then to store data according to appropriate representations, granularities, and quality. 
Service Management. The RWT can be directly integrated to the Web (in the case they have IP addresses or they are IPenabled when connected to the Internet) and be consequently able to understand each other through standardized web languages. They can also be integrated indirectly to the Web (e.g., sensor nodes in a WSN) for cost, energy and security considerations [3]. In both cases, the RWT should allow other devices to interoperate with them and mutually benefit from their services, which requires the abstraction of the RWT into reusable web services [5]. One or both of the W3C web service paradigms (REST-compliant Web services) and arbitrary Web services can be adopted.

The RWT services should be generated on-the-fly or at least within appropriate timeframes [5]. Although some technologies (e.g., FlyPort: www.openpicus.com) and research initiatives (e.g., [12]) have successfully embedded tiny web servers on mobile devices, additional research and development efforts are still needed, particularly because of the physical constraints of RTW. Furthermore, the services of RWT should be published in appropriate locations with convenient mechanisms for their discovery. In this regard, existing searching engines and algorithms must be reconsidered in order to allow an efficient and effective discovery of RWT services. Because of the limited capabilities of RWT, service composition could be a challenging solution where a group of RWT collaboratively create complex services from their individual elementary services. Furthermore, although the mobility of RWT offers new opportunities for service composition, it also brings new challenges, basically because it does not guarantee a durable availability of service providers.

Networking and Communication. Several technologies and standards have been proposed for smart things' communication, since many years. The sporadic mobility of RWT makes communication difficult, especially in the context of indoor applications. With the huge variety of types and manufacturers of RWT, interoperability is a growing concern. For instance, the RWT should be able to understand each other by using well-defined communication protocols. Since existing protocols, including UPnP and JXTA, have not been neither standardized nor widely accepted for embedded devices in industry, embedded tiny web servers could be an option [3]. The unpredictable mobility of RWT exacerbates the problems of their communication and urges the need for new lightweight protocols, where the identities, capabilities, and requirements of things are supported.

Trust, Privacy, and Security. The issues of security, privacy, and trust are always fueling intensive research works, especially within the context of large scale, open configurations in which specialized and non-specialized parties can participate anytime, anywhere. This is also the case for WoT where RWT can exchange and share data/services with other parties without necessary being firmly aware of their mutual intensions and actions. The option of embedding tiny web servers on RWT adds up additional security challenges. The use of REST-based interfaces makes it possible to have secure interactions using HTTP authentication or HTTPS [18]. However, the unpredictable configuration of the WoT and the lack of standards require new and revolutionary security mechanisms. The use of the social Web as a platform to ensure the trust and privacy of things has been advocated [19] to control Web-enabled things among trusted members on social Web sites [5]. However, additional research and development work is still needed for the success and widespread use of the WoT.

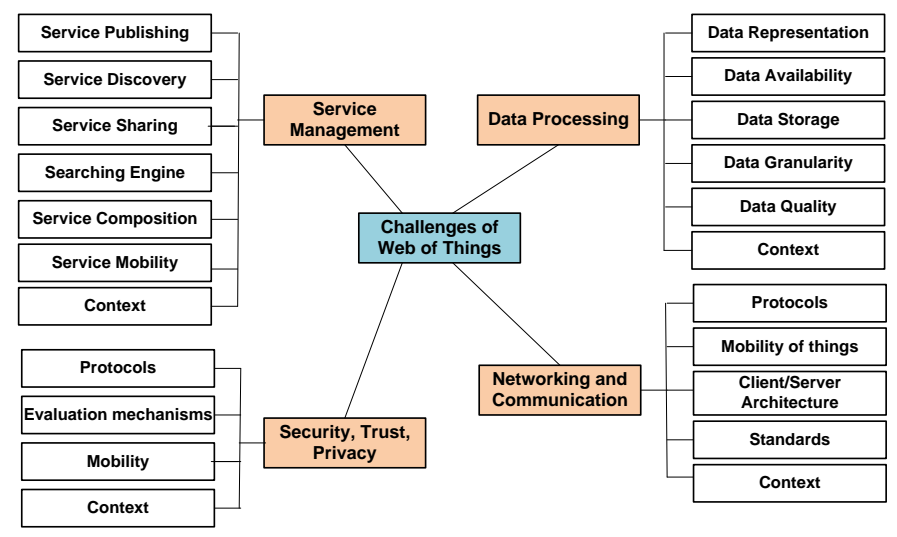

Fig.1. A proposed classification of the WoT challenges

\section{INTELLIGENT WEB OF THINGS}

In this section, we propose a multiagent-based architecture in order to deal with the challenges of WoT. This architecture is expected to be embedded on RWT. We particularly focus on the issue of service composition. We apply the proposed ideas to the scenario of Vehicular Ad-Hoc Networks.

\section{A. Need for Intelligence}

Because of their limited capabilities, unplanned mobility, non-standardized communication protocols, and potentially heterogeneous data formats, accuracy, and granularity, the spatially distributed RWT definitively need appropriate mechanisms to make convenient actions at the right time, depending on their current context and capabilities. In this paper, we argue that the multiagent system paradigm (MAS) could be appropriate for the WoT, thanks to its proven flexibility, autonomy, and intelligence to solve complex problems within highly dynamic, constrained, and uncertain environments [20]. We believe that several well established agent-based techniques could perfectly bring solutions to the deficiency and challenges of RWT highlighted in Section III.

\section{B. Multiagent-Based Architecture}

In this section we propose a MAS-based architecture to be embedded on RWT to handle the WoT challenges at different lefels. Our architecture (Figure 2) includes four main modules: Data Filtering Agent (DFA), Content Generation Agent (CGA), Networking and Communication Agent (NCA), and Security, Trust, and Privacy Agent (STPA). The DFA processes and analyzes the data collected by local sensing devices as well as data received from neighboring devices. Agent-based techniques for data filtering (e.g., [24]) and data mining (e.g., [23]) can be used. The CGA will then be able to create elementary services which will be published later. If a 
given service is requested by a tier, the RWT should use appropriate communication protocols (e.g., 6LowPAN, Zigbee, $\mathrm{WiFi}$ ) as well as appropriate communication pathways to respond and convey the service demanded. This tasks is performed by the agent NCA. The operation of the RWT is carried out according to specific security, trust, and privacy rules handled by the STPA. These rules will be updated based on accumulated experience and the envisioned WoT application. Our architecture also includes an agent-based system which will be used by appropriate RWT for service composition.

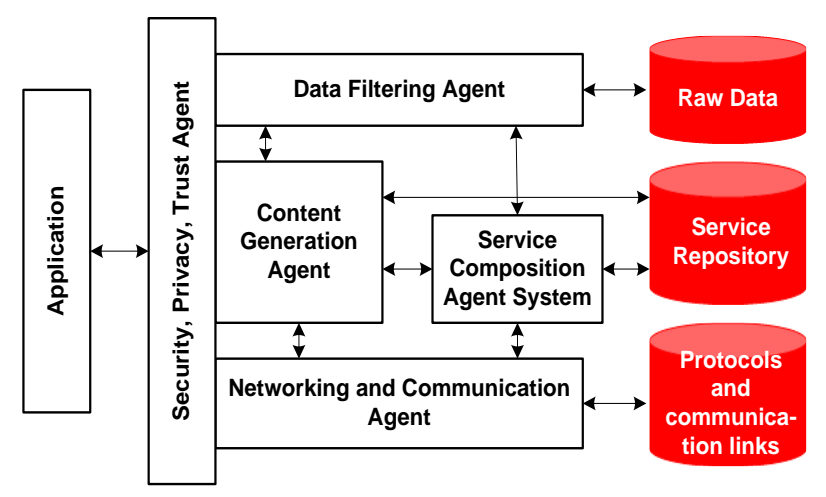

Fig. 2. An embedded multiagent architecture for RWT

\section{Service Composition}

When some required services cannot be provided individually, RWT should have the option to collaboratively generate new contents beyond their individual capabilities. This collaboration is particularly needed for energy and safety reasons as well as shortage of resources due to RWT mobility. In order to enable RWT collaboration, we propose to allow them creating clusters of things that we call Circles of Friends (CoF).

Each CoF will be composed of a group of RWT that will select each other based on their own preferences. Although the creation of CoFs is beyond the scope of this paper, we give a brief overview of how they are formed. Initially, while publishing its services, any RWT also publishes its wish to belong to a $\mathrm{CoF}$ with specific social and/or professional aims. Interested RWT could then contact each other to make a new CoF. One of the RWT is appointed as a Head of the CoF ( $\mathrm{HCoF})$. The $\mathrm{HCoF}$ is responsible of selecting the appropriate RWT to provide the currently requested services and make the necessary plans to generate complex services from elementary ones. In order to motivate RWT to join CoF so that complex services could be created more easily, smart things will be rewarded whenever they are participating and providing services within these circles. This will consequently affect their reputation in the WoT. A reward, and therefore a reputation, is also assigned to each $\mathrm{CoF}$ in order to motivate RWT to be active and maintain their CoFs.

In order to carry out the tasks of a $\mathrm{HCoF}$, any given RWT with appropriate physical resources will include a service composition agent system (see Figure 2) with the following agents: Translator Agent (TA), Service Generator Agent (SGA), Evaluator Agent (EA), and Executor Agent (XA) (Figure 3). The TA will receive the requests for services from its corresponding $\mathrm{CoF}$ and makes the necessary translations between the external languages and communication formats and the internal ones to the $\mathrm{HCoF}$. If the request cannot be understood then the $\mathrm{HCoF}$ can request the help of a member of the circle to make the necessary translations. Once the request is translated, specifications are sent to the SGA which will consult the repository of the services currently provided by the $\mathrm{CoF}$ as well as the currently active RWT and their rewards, trust, and security levels. Elementary services will be assigned to individual RWT. However, for complex services, the SGA plans and generates options to the EA. The agent EA will make the necessary assessments and selects an appropriate service composition plan with one backup plan. The selected plan will then be executed by the concerned RWT and monitored by the agent XA.

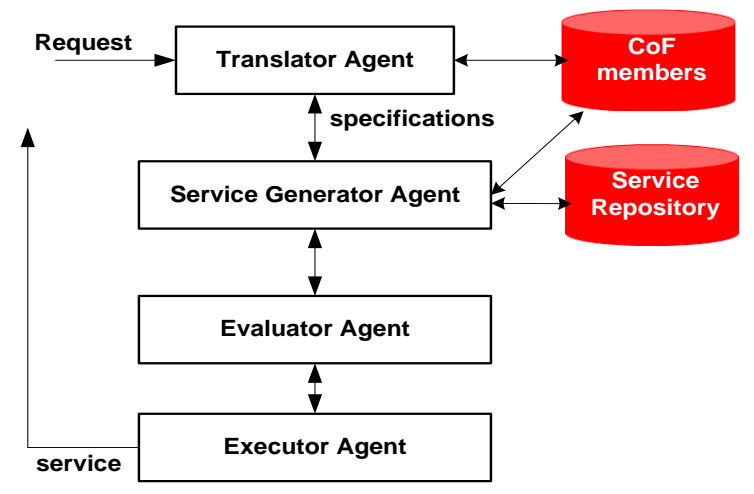

Fig. 3. Embedded multiagent system architecture for service composition

\section{INTELLIGENT WEB OF VEHICLES}

Cars are currently equipped with several sensing devices capable of acquiring real-time data about various onboard parameters, environmental conditions, and contextual events, allowing consequently a safer and more optimal commuters' travel times. Advances on sensing and communication facilities are impelling the evolution of the conventional Vehicular Ad-Hoc Networking (VANET) activities to the cloud, creating thereby the emergent notion of Internet of Vehicles (IoV) [21]. IoV includes networking infrastructures for Vehicle-to-Vehicle (V2V), Vehicle-to-Road (V2R), Vehicle-to-Human (V2H), and Vehicle-to-Sensor (V2S) interactions. In the IoV paradigm, each vehicle is potentially involved with heterogeneous devices, communication and networking technologies, service kinds, data formats/contents, accuracy/efficiency requirements, etc. In order to smoothly integrate and connect the RWT and information resources of the IoV along with a seamless integration with the social context, we coin the term Web of Vehicles (WoV) that particularly aims to leverage web protocols and technologies for VANET related devices/objects, while facilitating rapid service generation and sharing. Some of the devices on vehicles could be Web-enabled and could therefore be 
endowed with embedded tiny Web servers. These devices could play the role of proxies for other devices which cannot connect to the Internet. To this end, they may provide them with RESTful APIs for a direct Web-based access.

Within the context of WoV, let us suppose that a commuter wants to reduce his travel time between two given locations. In order to avoid unexpected traffic jams and reduce stoppage time at road intersections, a speed sensor on the commuter vehicle continuously reports information to an onboard decision unit (similar decision units could be embedded to any of the RWT in the WoV scenario). This unit also receives data from distance and environmental sensors as well as information/services from the road infrastructure, vehicles, humans, and sensors in the vicinity.

In addition to measuring the distance between the current vehicle and neighboring objects (vehicles, road infrastructures, etc.), a distance sensor on the commuter's vehicle could receive measurements from similar sensors on vehicles in the vicinity. These measurements should be cleaned and filtered by the distance sensor in order to assess the position of the vehicle with respect to its neighboring objects from the side where the sensor is deployed. The sensor should also timely share useful information with other appropriate RWT on the road. For a better assessment of the situation, all distance sensors on the commuter's vehicle will collect similar data and submit reports to the decision unit onboard. Agent-based techniques (e.g., $[23,24])$ could then be used for data filtering and mining purposes on any of the sensors/RWT.

As road safety is a shared matter, on-road vehicles have to accommodate each other and mutually exchange contextual information and services on-time. Examples of services may include vehicle driving conditions (speeding, planned driving directions, alerts on vehicle about critical situations, etc.), onroad events (traffic jams, accidents, etc.), and professional services (healthcare if the driver is doctor/nurse, plumber, etc.). The vehicles of the WoV will create CoFs. A CoF does not necessary consist of geographically collocated vehicles. For instance, some vehicles may share the same destination or the same social interests and therefore would like to maintain their $\mathrm{CoF}$, although they may be very far from each other because of traffic conditions.

For each CoF, one vehicle will be elected as $\mathrm{HCoF}$ using an appropriate clustering technique [27]. This vehicle will maintain the list of services provided by each of the vehicles in the circle. It can also request services on their behalf and enable them to socially connect with similar vehicles from other circles. The HCoF should always stay tuned to the needs of the members of the circle, update their rewards, plan the composition of complex services, etc. To this end, all requests received by the $\mathrm{HCoF}$ will be translated, when needed, into the internal language and formats by an onboard intelligent agent. Service composition will be planned by a special agent based on the current offering, trust, and capabilities of the vehicles in the CoF. Since some vehicles would be competing to offer their services and increase their rewards, an agent evaluator will fairly and carefully check service composition plans before handing over the approved plan to an executor agent to monitor the required actions. Rewards and trust levels will then be updated accordingly once this plan is achieved.

\section{CONCLUSION}

The work we presented in this paper is a first conceptual step towards enabling Intelligent WoT (IWoT) through the use of multiagent system techniques. A lot of issues still need to be addressed and explored in the future to fully implement our vision. In this regard, the multiagent-based architecture proposed for service composition needs to be refined, implemented and experimented. Then it needs to be extended to address the other challenges presented in the paper, including Data Processing and Storage, Networking and Communication, and Trust, Privacy, and Security.

\section{REFERENCES}

[1] CASAGRAS (2009). CASAGRAS Final Report: RFID and the Inclusive Model for the Internet of Things, 2009, pp. 10-12

[2] Pang, Z. (2013). Technologies and Architectures of the Internet-of-Things (IoT) for Health and Well- being. KTH Royal Institute of Technology, ISBN 978-91-7501-736-5

[3] Zeng, D., Guo, S., Cheng, Z. (2011). The Web of Things: A Survey (Invited Paper), J. Communications, vol. 6, no. 6, pp. 424-438, September 2011

[4] Cheshire, S., Steinberg, D.H. (2005). Zero Configuration Networking, the Definitive Guide, O’Reilly, 2005

[5] Mathew, S.S., Atif, Y., Sheng, Q.Z., Maamar, Z. (2013). Internet of Things and Inter-cooperative Computational Technologies for Collective Intelligence, 2013 / Bessis, N., Xhafa, F., Varvarigou, D., Hill, R., Li, M. (ed./s), pp.1-23

[6] T. Berners-Lee (2008). The Web of Things, Special theme on the future web. ERCIM News - the European Research Consortium for Informatics and Mathematics, 2008. http://ercim-news.ercim.eu/en72/keynote, visited: 03/09/2015

[7] Raggett, D. (2010). The Web of Things: Extending the Web into the Real World, SOFSEM 2010: Theory and Practice of Computer Science, Jan 2010

[8] B. Ostermaier, M. Kovatsch, and S. Santini, Connecting Things to the Web using Program-mable Low-power WiFi Modules, In the proceedings of the 2nd International Workshop on the Web of Things (WoT 2011), 2011

[9] D. Guinard and V. Trifa, Towards the Web of Things: Web Mashups for Embedded Devices, In the proceedings of the workshop Mashups, Enterprise Mashups and Lightweight Composi-tion on the Web (MEM'09), 2009

[10] R. T. Fielding, Architectural styles and the design of networkbased software architectures, Ph.D. dissertation, 2000

[11] C. Pautasso, O. Zimmermann, and F. Leymann, Restful web services vs. 'big' web services: making the right architectural decision, In the proceedings of the 17th international conference on World Wide Web, ser. WWW '08. New York, NY, USA: ACM, pp. 805-814, 2008

[12] S. Duquennoy, G. Grimaud, and J.J. Vandewalle, The Web of Things: Interconnecting Devices with High Usability and Performance, In the proceedings of the International confer-ence on Embedded Software and Systems (ICESS'09), 2009

[13] G. Kortuem, F. Kawsar, V. Sundramoorthy, D. Fitton. (2010) Smart Objects as Building Blocks for the Internet of Things. Proc. Of the IEEE Internet Computing, vol. 14, n. 1, pp. 44-51 
[14] Angulo-Lopez, P., Jiménez-Pérez, G. (2012). Collaborative Agents Framework for the Internet of Things. Intelligent Environments (Workshops) 2012: 191-199

[15] A. M. Mzahm, M. S. Ahmad, Alicia Y. C. Tang, Agents of Things (AoT): An Intelligent Operational Concept of the Internet of Things (IoT), 13th International Conference on Intelligent Systems Design and Applications (ISDA 2013), pp. $159-164,2013$

[16] Zhong, N., Ma, J., Huang, R., Liu, J., Yao, Y.Y., Zhang, Y., Chen, J. (2010). Research Challenges and Perspectives on Wisdom Web of Things (W2T), Journal of Supercomputing, Springer. DOI 10.1007/s11227-010-0518-8 (2010)

[17] Sawyer, P., Pathak, A., Bencomo, N., Issarny, V. (2012) How the Web of Things Challenges Requirements Engineering, 3 rd Workshop on The Web and Requirements Engineering at 12th International Conference on Web Engineering ICWE 2012, Berlin Germany, 27th July 2012

[18] E. Wilde, Putting Things to REST, UCB iSchool Report 2007015, School of Information, UC Berkeley, 2007

[19] D. Guinard, M. Fischer, and V. Trifa, Sharing Using Social Networks in a Composable Web of Things, In the proceedings of the 1st IEEE International Workshop on the Web of Things (WoT 2010) at IEEE PerCom, Germany, 2010

[20] Bandyopadhyay S., Coyle E.J. (2013). An energy efficient hierarchical clustering algorithm for wireless sensor networks", Proc. of INFOCOM 20013, IEEE Societies, 2013, vol. 3, pp. 1713-1723

[21] Gerla, M.; Eun-Kyu Lee; Pau, G.; Uichin Lee, (2014) Internet of vehicles: From intelligent grid to autonomous cars and vehicular clouds, in Internet of Things (WF-IoT), 2014 IEEE World Forum on , vol., no., pp.241-246, 6-8 March 2014

[22] Shelby, Z.: Embedded web services. IEEE Wireless Communication Magazine 17(6), 52-57 (2010)

[23] K. A. Albashiri, (2010) "An investigation into the issues of Multi-Agent Data Mining", Ph.D. dissertation, The University of Liverpool, Ashton Building, Ashton Street, Liverpool L69 3BX, United Kingdom

[24] Skocir, P., Maracic, H., Kusek, M., Jezic, G. (2015). Data Filtering in Context-Aware Multi-Agent System for Machine-toMachine Communication, G. Jezic et al. (ed.), Agent and MultiAgent Systems: Technologies and Applications, Smart Innovation, Systems and Technologies 38, DOI 10.1007/978-3319-19728-9_4

[25] Rajkumar, R., Lee, I., Sha, L., Stankovic, J. (2010). Cyberphysical systems: the next computing revolution. In Proceedings of the 47th Design Automation Conference. ACM, New York, NY, USA, pp. 731-736

[26] Jabeur, N., Sahli, N., Zeadally, S. (2014)., ABAMA: An AgentBased Architecture for Mapping Natural Ecosystems onto Wireless Sensor Networks, Invited Paper, in Proceedings of 9th International Conference on Future Networks and Communications (FNC-2014), Elsevier Procedia Computer Science, Volume 34, Canada, August 2014 doi:10.1016/j.procs.2014.07.020

[27] Vodopivec, S.; Bester, J.; Kos, A., "A survey on clustering algorithms for vehicular ad-hoc networks," in Telecommunications and Signal Processing (TSP), 2012 35th International Conference on , vol., no., pp.52-56, 3-4 July 2012 